\title{
Effects of primary and recurrent sacral chordoma on the motor and nociceptive function of hindlimbs in rats: an orthotopic spine model
}

\author{
*Rachel Sarabia-Estrada, PhD,, Alejandro Ruiz-Valls, MD, ${ }^{2}$ Sagar R. Shah, PhD,, \\ A. Karim Ahmed, BS, ${ }^{2}$ Alvaro A. Ordonez, MD, ${ }^{3}$ Fausto J. Rodriguez, MD, ${ }^{4}$ \\ Hugo Guerrero-Cazares, PhD, ${ }^{1}$ Ismael Jimenez-Estrada, PhD, ${ }^{7}$ Esteban Velarde, BS, ${ }^{5}$ \\ Betty Tyler, BA, ${ }^{2}$ Yuxin Li, MD, ${ }^{9}$ Neil A. Phillips, MSci, ${ }^{2}$ C. Rory Goodwin, MD, PhD, ${ }^{2}$ \\ Rory J. Petteys, MD, ${ }^{2}$ Sanjay K. Jain, MD, ${ }^{3}$ Gary L. Gallia, MD, PhD, ${ }^{2}$ Ziya L. Gokaslan, MD, ${ }^{8}$ \\ Alfredo Quinones-Hinojosa, MD, ${ }^{1}$ and Daniel M. Sciubba, MD² \\ 'Department of Neurologic Surgery, Mayo Clinic, Jacksonville, Florida; Departments of ${ }^{2}$ Neurosurgery and ${ }^{3}$ Pediatrics, \\ Center for Infection and Inflammation Imaging Research, Departments of ${ }^{4}$ Pathology-Neuropathology, ${ }^{5}$ Radiation Oncology \\ and Molecular Radiation Sciences, and ${ }^{\circ}$ Biomedical Engineering, Johns Hopkins University School of Medicine, Baltimore, \\ Maryland; ' ${ }^{7}$ Department of Physiology, Biophysics, and Neurosciences, Research Center for Advanced Studies IPN, Mexico City, \\ Mexico; ${ }^{8}$ Department of Neurosurgery, The Warren Alpert Medical School of Brown University, Providence, Rhode Island; and \\ 9Department of Neurosurgery, Jinan General Hospital of PLA, Jinan, China
}

\begin{abstract}
OBJECTIVE Chordoma is a slow-growing, locally aggressive cancer that is minimally responsive to conventional chemotherapy and radiotherapy and has high local recurrence rates after resection. Currently, there are no rodent models of spinal chordoma. In the present study, the authors sought to develop and characterize an orthotopic model of human chordoma in an immunocompromised rat.
\end{abstract}

METHODS Thirty-four immunocompromised rats were randomly allocated to 4 study groups; 22 of the 34 rats were engrafted in the lumbar spine with human chordoma. The groups were as follows: UCH1 tumor-engrafted $(n=11), J H C 7$ tumor-engrafted $(n=11)$, sham surgery $(n=6)$, and intact control $(n=6)$ rats. Neurological impairment of rats due to tumor growth was evaluated using open field and locomotion gait analysis; pain response was evaluated using mechanical or thermal paw stimulation. Cone beam CT (CBCT), MRI, and nanoScan PET/CT were performed to evaluate bony changes due to tumor growth. On Day 550, rats were killed and spines were processed for $\mathrm{H} \& \mathrm{E}$-based histological examination and immunohistochemistry for brachyury, $\mathrm{S} 100 \beta$, and cytokeratin.

RESULTS The spine tumors displayed typical chordoma morphology, that is, physaliferous cells filled with vacuolated cytoplasm of mucoid matrix. Brachyury immunoreactivity was confirmed by immunostaining, in which samples from tumor-engrafted rats showed a strong nuclear signal. Sclerotic lesions in the vertebral body of rats in the UCH1 and JHC7 groups were observed on CBCT. Tumor growth was confirmed using contrast-enhanced MRI. In UCH1 rats, large tumors were observed growing from the vertebral body. JHC7 chordoma-engrafted rats showed smaller tumors confined to the bone periphery compared with $\mathrm{UCH} 1$ chordoma-engrafted rats. Locomotion analysis showed a disruption in the normal gait pattern, with an increase in the step length and duration of the gait in tumor-engrafted rats. The distance traveled and the speed of rats in the open field test was significantly reduced in the $\mathrm{UCH} 1$ and $\mathrm{JHC} 7$ tumor-engrafted rats compared with controls. Nociceptive response to a mechanical stimulus showed a significant $(p<0.001)$ increase in the paw withdrawal threshold (mechanical hypalgesia). In contrast, the paw withdrawal response to a thermal stimulus decreased significantly $(p<0.05)$ in tumor-engrafted rats.

CONCLUSIONS The authors developed an orthotopic human chordoma model in rats. Rats were followed for 550 days using imaging techniques, including MRI, CBCT, and nanoScan PET/CT, to evaluate lesion progression and bony integrity. Nociceptive evaluations and locomotion analysis were performed during follow-up. This model reproduces cardinal signs, such as locomotor and sensory deficits, similar to those observed clinically in human patients. To the authors'

ABBREVIATIONS CBCT = cone beam computed tomography.

SUBMITTED September 16, 2016. ACCEPTED December 30, 2016.

INCLUDE WHEN CITING Published online June 9, 2017; DOI: 10.3171/2016.12.SPINE16917.

* Drs. Sarabia-Estrada, Ruiz-Valls, and Shah contributed equally to this work. Drs. Sarabia-Estrada and Sciubba share senior authorship of this work. 
knowledge, this is the first spine rodent model of human chordoma. Its use and further study will be essential for pathophysiology research and the development of new therapeutic strategies.

https://thejns.org/doi/abs/10.3171/2016.12.SPINE16917

KEY WORDS chordoma; spine; tumor; model; locomotion; nociception; rat; oncology

$\mathrm{C}$ HORDOMA is a tumor of the spinal column with an incidence of 0.08 cases per 100,000 people and a median survival of 6.29 years. ${ }^{27}$ These characteristically slow-growing and locally invasive tumors most commonly present in the sacrococcygeal region $(50 \%-$ $60 \%$ ), followed by the clivus/spheno-occipital region $(15 \%-35 \%)$ and mobile spine $(15 \%)^{7,42}$ Clival chordomas tend to cluster in a younger population, whereas sacrococcygeal chordomas present more frequently in older adults. ${ }^{16,29,50}$ The clinical presentation of patients suffering from chordoma is dependent on tumor location, and pain is the most common presenting symptom. ${ }^{20}$ Patients with clival chordoma frequently present with cranial nerve palsies and headaches, whereas patients with sacral chordoma commonly present with sacral or flank pain and gait disturbances. ${ }^{29,50}$

Chordomas seldom metastasize, but when they do, the distal lesions occur late in the course of the disease (rates of chordoma metastasis reported in the literature range from $5 \%$ to $43 \%)^{7,11,28,48,51}$ The current standard of care includes a combination of resection and radiotherapy..$^{13,14,33}$ However, due to the aggressive pattern of local invasion and risks to adjacent vital structures, complete tumor resection is balanced against the potential risks of neurological deficits and the potential for a high incidence of recurrence. ${ }^{27}$ The major cause of death in patients with chordoma is associated with local aggressiveness. ${ }^{2}$

Despite the evolution of surgical techniques and advancements in medical and radiotherapies, the development of therapeutic strategies to combat this disease is limited by the availability of orthotopic in vivo models that recapitulate the histological and clinical features of chordoma. In 2014, Burger et al. developed a novel zebrafish model of chordoma driven by notochordal-specific green fluorescent protein-tagged HRASV12 expression. Their model is histologically comparable to human chordoma and has a rapid onset, which is well suited for preliminary drug screening. 6 However, development of a clinically relevant spine chordoma model to study the surgical and clinical effects on this tumor in a human xenograft and mammalian-based system would increase our understanding of the biology of these rare malignancies and allow for the translation of potential therapies to human patients. Thus, it is of utmost interest to develop a tumor model system that closely resembles the etiology and pathophysiology of the disease in patients.

To this end, we present an orthotopic human chordoma rat model that replicates the histological and clinical features present in patients. We used 2 patient-derived primary and recurrent chordoma cell lines (UCH1 and JHC7, respectively) to characterize the development of intraspinal chordomas in rats. Following tumor implantation, imaging studies and neurological evaluation were conducted to assess nervous system dysfunction in the form of pain responses and behavioral changes. Our study will allow researchers to test experimental therapy strategies in a clinically relevant system, thus providing predictive models of treatment efficacy.

\section{Methods}

\section{Animal Procedure}

All procedures were performed according to the Guide for the Care and Use of Laboratory Animals and were approved by the Animal Care and Use Committee (Johns Hopkins University). Forty adult female, 5-weekold, immunocompromised rats (Cr:NIH-RNU, Charles River Laboratories) were maintained in standard cages in groups of 2 or 3 in a controlled colony room, under standard environmental conditions, with free access to food and water. The animals were randomly allocated to different experimental groups.

\section{Human Chordoma Cell Lines}

We used 2 primary-cultured human chordoma cell lines: UCH1 and JHC7. UCH1 is a human chordoma cell line established from a recurrent sacral chordoma (ATCC). UCH1 cells were cultured and expanded using Iscove's (Roswell Park Memorial Institute medium [4:1] + $10 \%$ fetal bovine serum) with additional $1 \%$ L-glutamine. ${ }^{39}$ The JHC7 cell line was derived from clinical specimens obtained from a female patient with primary sacral chordoma who had no history of treatment for oncological disease. ${ }^{19}$ The JHC7 chordoma cell line was maintained using MesenPRO RS medium + MesenPRO RS Supplement (Thermo Fisher Scientific), GlutaMAX 1×, and antibiotic and antimycotic $1 \times$. Cells were maintained in a humidity incubator in an atmosphere of $5 \% \mathrm{CO}_{2}$ at $37^{\circ} \mathrm{C} . .^{19}$

\section{Xenograft Establishment}

To establish a subcutaneous chordoma, JHC7 or UCH1 cells were trypsinized and centrifuged at $180 \mathrm{~g}$ for $5 \mathrm{~min}$ utes at $4^{\circ} \mathrm{C}$. Cells were counted and $6 \times 10^{6}$ cells were resuspended in their respective media mixed with Growth Factor Reduced Matrigel (Corning) at a 2:1 ratio, to a final volume of $200 \mu \mathrm{l}$. The cell suspension was injected subcutaneously into the flank of anesthetized rats $(n=3$ for each cell line, Fig. 1A). Rats were maintained in vivarium conditions until a detectable tumor was appreciated (approximately 6-8 months).

\section{Establishment of Chordoma in the Lumbar Spine}

After flank tumors were established, the lesion was excised and either partially processed for histopathological analysis or used to establish the orthotopic chordoma model. To establish an orthotopic chordoma model in the lumbar spine, 34 female immunocompromised rats (5 weeks old) were randomly allocated into 4 surgical groups: 1) UCH1 tumor-engrafted $(\mathrm{n}=11)$, 2) JHC7 tu- 
A
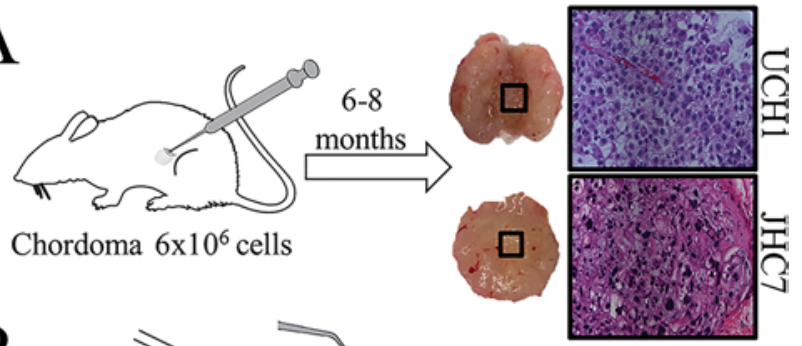

$\mathrm{B}$ Chordoma $6 \times 10^{6}$ cells
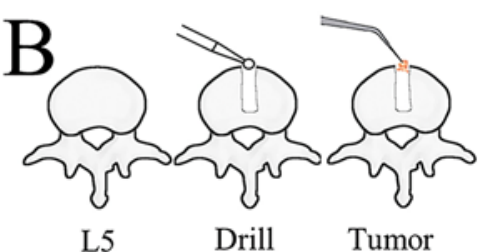

Tumor implant seal
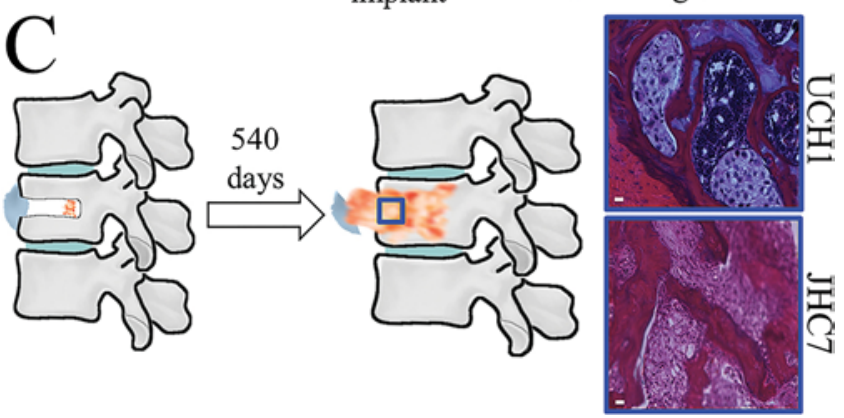

FIG. 1. Human orthotopic chordoma model in the spine of immunocompromised rats. A: $\mathrm{UCH} 1$ or $\mathrm{JHC} 7$ cells were injected in the flank of 5-week-old female rats. After 6-8 months, subcutaneous tumors showed a multilobulated morphology, and physaliferous cells were observed between the tissues in the flank. $\mathrm{H}$ \& $\mathrm{E}$ sections, original magnification $\times 10$; calibration bars $=20 \mu \mathrm{m}$. B: Illustrations of the engrafting procedure in the $L-5$ vertebral body with subcutaneous tumor tissue from donor rats. C: After 540 days from tumor engrafting, chordomas invaded the vertebral body in the $\mathrm{UCH} 1$ and $\mathrm{JHC} 7$ rats. Bone tissue with tumor invasion showed the typical physaliferous cells, with intracytoplasmic vacuolization in the $\mathrm{H} \& \mathrm{E}$ sections (original magnification $\times 20$; calibration bars $=0.02 \mu \mathrm{m})$. Figure is available in color online only.

mor-engrafted $(n=11), 3)$ sham surgery $(n=6)$, and 4) naïve control $(n=6)$ rats. We performed an abdominal surgical approach to the L-5 vertebral body as previously described. ${ }^{26}$ Briefly, while the rat was under complete anesthesia, the L-5 vertebral body was drilled $0.5 \mathrm{~mm}$ superior to the intervertebral disc to create a cavity for tumor implantation. Tumor samples $\left(0.1 \mathrm{~cm}^{3}\right)$, obtained from the subcutaneous chordoma xenografts, were deposited in the cavity and then sealed with Geristore dual-cure resin ionomer polymer (DenMat) (Fig. 1B). The sham surgery group underwent an identical surgical approach with the exception that no tumor was implanted. The abdominal cavity was closed, and animals were maintained in vivarium conditions. Interestingly, when a chordoma cell suspension in Matrigel/serum-free media solution was injected directly into the vertebral body, no tumor growth was detected 8 months after implantation (data not shown).

\section{Behavioral Tests}

Motor and nociceptive functions were evaluated at Days 530 and 535, respectively, after tumor implantation to determine the effects of the chordoma growth at a functional level. For all behavioral evaluations, the rats were gently handled for 5 minutes during a 5-day period to minimize stress. All of the behavioral tests were performed in a quiet, red-illuminated room and were done between 1200 and 1700 hours by a blinded researcher to avoid bias in the results.

\section{Kinematic Analysis of Gait Locomotion}

Rat locomotion was video recorded and evaluated as previously described. ${ }^{24,38}$ Briefly, each rat was trained to walk in a narrow Plexiglas runway to ensure locomotion in a straight line. Rats' hip, knee, and ankle (that is, lateral malleolus) joints were marked. Unrestrained gait of nontumor and tumor-engrafted rats was recorded at 30 frames per second. We recorded 3- to 4-stride cycles per rat. Video analysis was performed using ImageJ software (http:// rsb.info.nih.gov/ij/; National Institutes of Health) followed by our proprietary Walking Rats Software (developed by the IJ-E Laboratory).

Joint angles, stride length, duration, and flexion-extension of each articulation were determined. Swing and stance phases, as well as the hip-knee-ankle pendulumlike movement of each step cycle, were identified as described previously. ${ }^{36}$ Briefly, the marked points in the rat's hindlimb were tracked frame by frame, obtaining 2D coordinates ( $\mathrm{x}$ and $\mathrm{y}$ ) using ImageJ software. Next, data were imported into Microsoft Office Excel and further analyzed with preassembled Excel sheets to model body segments as rigid straight lines between the marked points.

The kinematics of gait was then reconstructed from changes in the marked points located between consecutive frames, facilitating the generation of stick diagrams (superimposing modeled body segments of every frame) and spatial displacement plots. Angles and distances could be calculated directly by the software. Optical deformation of the image produced by the camera lens was determined and corrected by using an acrylic square $(5 \times 5 \mathrm{~cm})$, which served as a bidimensional scale. ${ }^{34}$

\section{Open Field Test for Motor Behavior}

To evaluate free rat movement, we used an $\mathrm{OF}-3 \mathrm{C}$ (Bioseb) with a previously published method of open field analysis. ${ }^{34}$ The open field test was performed in a square (length $1000 \mathrm{~mm} /$ width $1000 \mathrm{~mm} /$ height $400 \mathrm{~mm}$ ) arena with gray acrylic walls. Rats began the test at the center of the arena and were given 5 minutes to freely explore. The total distance traveled $(\mathrm{cm})$ and the mean speed $(\mathrm{cm} / \mathrm{sec})$ in the arena were automatically registered.

\section{Mechanical Paw Withdrawal Threshold Test}

Nociceptive response was evaluated using the rodent pincher analgesia meter (Part \# 2450, IITC Life Science, Inc.) to apply pressure on the left hind paw. The system measures and displays the amount of force (in grams) that has been applied to the rat's paw. ${ }^{47}$ Response to the pressure applied (in grams) was manually registered upon paw withdrawal from stimulus. Pressure was increased by 5 gram/sec increments and measurements were performed in triplicates. Researchers performing the test in all groups were blinded to avoid experimenter bias. 


\section{Thermal Paw Withdrawal Latency Test}

To evaluate the magnitude of thermal hyperalgesia, the thermal plantar (Hargreaves method) testing device was used to measure hind paw withdrawal latency in response to radiant heat (Plantar Test Instrument, Ugo Basile), as described elsewhere. ${ }^{17}$ Briefly, each rat was placed in a transparent Plexiglas chamber and given 5 minutes to habituate. An infrared heat source was positioned under the glass floor and directly inferior to the plantar surface of the hind paw. The infrared heat source $\left(50^{\circ} \mathrm{C}\right)$ was subsequently activated with an automatic timer for rat reaction. Rat paw withdrawal deactivated the infrared source and the duration of stimulus was automatically recorded. A predetermined cutoff was established at 20 seconds to prevent tissue damage. The average withdrawal latencies for the left hind paw were determined from the average of 3 trials separated by a 10-minute interval to prevent thermal sensitization.

\section{In Vivo Imaging of Spine Chordoma}

Rats engrafted with chordoma into the spines were imaged by cone beam computed tomography (CBCT) at Day 450 after tumor implantation, using a small animal research radiation platform (Xstrahl Life Sciences) as previously described. ${ }^{49,52}$ Images were acquired at 65 $\mathrm{kVp}$ and $0.7 \mathrm{~mA}$ using a $20 \times 20-\mathrm{cm}$ beam. CBCT images were used to identify bone lesions in the UCH1 and JHC7 rats after tumor engraftment. Contrast-enhanced MRI was performed on Day 540. Live rats in the experimental groups (UCH1 and JHC7) were administered gadopentetate dimeglumine $(0.1 \mathrm{mmol} / \mathrm{kg}$ intramuscular, Magnevist). Using a 9.4-T MRI animal scanner (BioSpec Bruker Series, Bruker Corporation), T1- and T2-weighted sequences were obtained before and after contrast administration. On Day 545, tumor-engrafted rats (1 rat per experimental group) underwent nanoScan PET/CT within a sealed biocontainment device (Minerve) as described previously ${ }^{32}$ and then were imaged using a small animal imager (Mediso). Images were reconstructed and visualized using VivoQuant 2.50 (inviCRO) and 3D images were obtained from contoured 2D images.

\section{Histopathological Analysis}

Rats engrafted with chordoma cells into the spine were killed on Day 550 after tumor engrafting. Rats were intracardially perfused with $4 \%$ paraformaldehyde; then spines were harvested and postfixed for 12 hours. After fixation, spines were decalcified with hydrochloric acid for 8 hours. A second dissection was made to localize the area where the tumor was engrafted. The segments were then processed for dehydration, clearing, and infiltration with paraffin. Axial sections of tissue $(10 \mu \mathrm{m}$ thick) were obtained and stained with $\mathrm{H} \& \mathrm{E}$. An independent blinded pathologist evaluated the tissue characteristics.

\section{Immunostaining Procedures}

A separate group of tissue sections (10 $\mu \mathrm{m}$ thick) were immunostained for brachyury (Santa Cruz Biotechnology, C19, sc-17745, dilution 1:50), S100 $\beta$ (Abcam, ab11178, dilution 1:500), and cytokeratin (Keratin-Pan AE1/AE3,
Thermo Scientific, MS-343-P, dilution 1:100), as described previously. ${ }^{19}$ Biotinylated secondary antibodies and streptavidin-conjugated horseradish peroxidase were used to visualize the label. The chromogen 3'3-diaminobenzidine was used to visualize immunoreactivity. An independent blinded pathologist analyzed the stained sections.

\section{Statistical Analysis}

Locomotion and nociceptive parameters were evaluated using a nonparametric 1-way ANOVA, followed by a Dunnett post hoc test. Statistical significance was determined using GraphPad Prism version 6.0 software. The alpha value was set at 0.05 . Results are reported as the mean \pm SEM.

\section{Results}

\section{Xenograft Characteristics in the Flank}

Six months after the subcutaneous injection of UCH1 and JHC7 chordoma cells in the flank, rats developed small oval-shaped tumors that reached $5.69 \mathrm{~cm}^{3}$ and $2.86 \mathrm{~cm}^{3}$, respectively. Upon gross macroscopic evaluation, these tumors showed a lobular growth pattern with a peripheral pseudocapsule. Cross-sectional examination showed pale fibrous or fleshy areas mixed with mucinous matrix. Histologically, $\mathrm{H} \& \mathrm{E}$-stained tumor sections showed clusters of small round nuclei with large, partly vacuolated-appearing physaliferous cytoplasm, consistent with the classically described histology of chordoma and phenotypically similar to the histopathology of the original patient tumor (Fig. 1A).

\section{Growth of Human-Derived Chordoma in the Vertebral Body of Rats}

Examination of all rats that underwent tumor implantation showed tumor growth in $54.54 \%$ of UCH1 (recurrent sacral chordoma) and $81 \%$ of JHC7 (primary sacral chordoma) at 550 days postimplantation. Macroscopically, UCH1-engrafted rats developed large tumor masses emerging from the vertebral body into the abdominal cavity, whereas JHC7 tumors were comparatively smaller with similar distribution. Tumors did not invade other surrounding soft tissues or organs. Bladder and bowel functions were conserved until the animals were euthanized. After gross sectioning, we observed tumor anatomy similar to that described for the subcutaneous tumors, with a pseudocapsulated lobular pattern and mixed fibrous and fleshy areas. In the vertebral body, tumor was localized to the site of implantation (Fig. 2A and B). The adjacent bone was noted to be fragile and soft. We observed that a single vertebral body was affected by the chordoma in both groups.

Histopathological analysis revealed cords and strands of tumor cells in an abundant myxoid matrix with mild atypia. Small areas of the vertebral body were composed of sheets of adipocyte-like cells, without a nodular growth pattern or myxoid background. Analysis of the vertebral bodies demonstrated proliferation of adipocyte-like vacuolated cells between thickened trabecular frameworks (Fig. 2C) with intercellular myxoid matrix, nuclear atypia, and proliferation of physaliferous cells with a prominent 


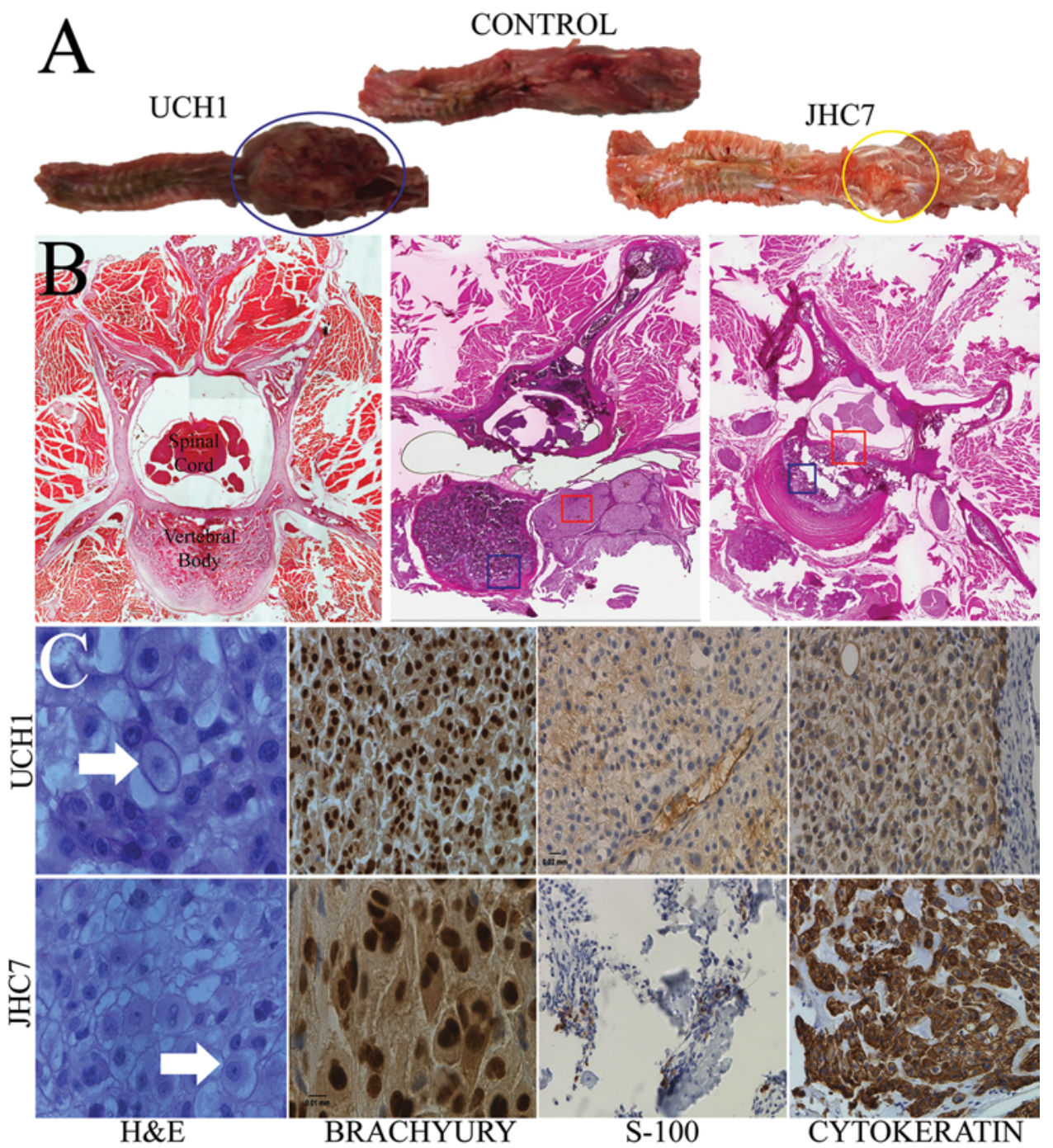

FIG. 2. Histopathology of $\mathrm{UCH} 1$ and JHC7 spine chordomas. A: Macroscopic morphology of 1 representative rat each from the $\mathrm{UCH} 1, \mathrm{JHC7}$, and control groups. Gross morphology examination of the L-5 vertebra showed a pink-yellow tissue encapsulating the L-5 vertebral body in the tumor-implanted UCH1 (blue solid line) and JHC7 (yellow solid line) rats. The UCH1 rat had a big mass emerging from the vertebral body, and the $\mathrm{JHC} 7$ rat presented with a small bone overgrowth in the vertebral body. B: Histological examination $(\mathrm{H} \& \mathrm{E}$ sections, original magnification $\times 2.5)$ of the vertebrae axial sections confirmed chordoma (red square) emerging from the vertebral bodies without spinal cord involvement in the UCH1 tumor-engrafted rats (blue square). JHC7 rats presented with a small extradural chordoma (red square) and a tumor emerging from the vertebral body (blue square). No histopathological changes were observed in the control rats. C: $\mathrm{H}$ \& E stain of the chordoma samples showed physaliferous cells (white arrows) with intracytoplasmic vacuolization in the spine chordomas formed by $\mathrm{UCH} 1$ and $\mathrm{JHC} 7$ cell lines, strong nuclear immunoreactivity for brachyury, and positive immunoreactivity for S100ß. Cytokeratins were observed in the UCH1 and JHC7 tumor-implanted groups. Original magnification $\times 63$; calibration bars $=0.01 \mathrm{~mm}$. Figure is available in color online only.

intracellular eosinophilic matrix, consistent with tumor infiltration. Tumor cells were positive for brachyury, cytokeratin, and S100 $\beta$, as demonstrated by immunohistochemistry. Prominent nuclear immunoreactivity of brachyury, as well as cytoplasmic expression of cytokeratin, was observed.

\section{Imaging Features of Orthotopic Human Chordoma in Rats}

CBCT for UCH1- and JHC7-bearing rats demonstrated the presence of chordomas in the spine. All rats that developed intraspinal tumors also developed a kyphotic deformity in the thoracolumbar spine (Fig. 3A and B). Lesions were identified in the L-5 vertebral body of tumor- engrafted rats. Trabecular sclerosis and osteolysis, which are 2 radiological findings commonly observed in human patients suffering from chordoma, were present in the adjacent vertebral bodies (Fig. 3B and G, black asterisks). No imaging alterations were observed in the naïve control and sham groups.

Imaging of soft tissues with contrast-enhanced MRI showed contrast enhancement in both the UCH1 and JHC7 groups. There was a significant extension of the tumor mass into the paravertebral space (Fig. $3 \mathrm{C}$ and $\mathrm{H}$ ) and compression of the spinal cord. In the UCH1 group, the scan showed a heterogeneous enhancement of the tumor mass, with high signal intensity emerging from the verte- 

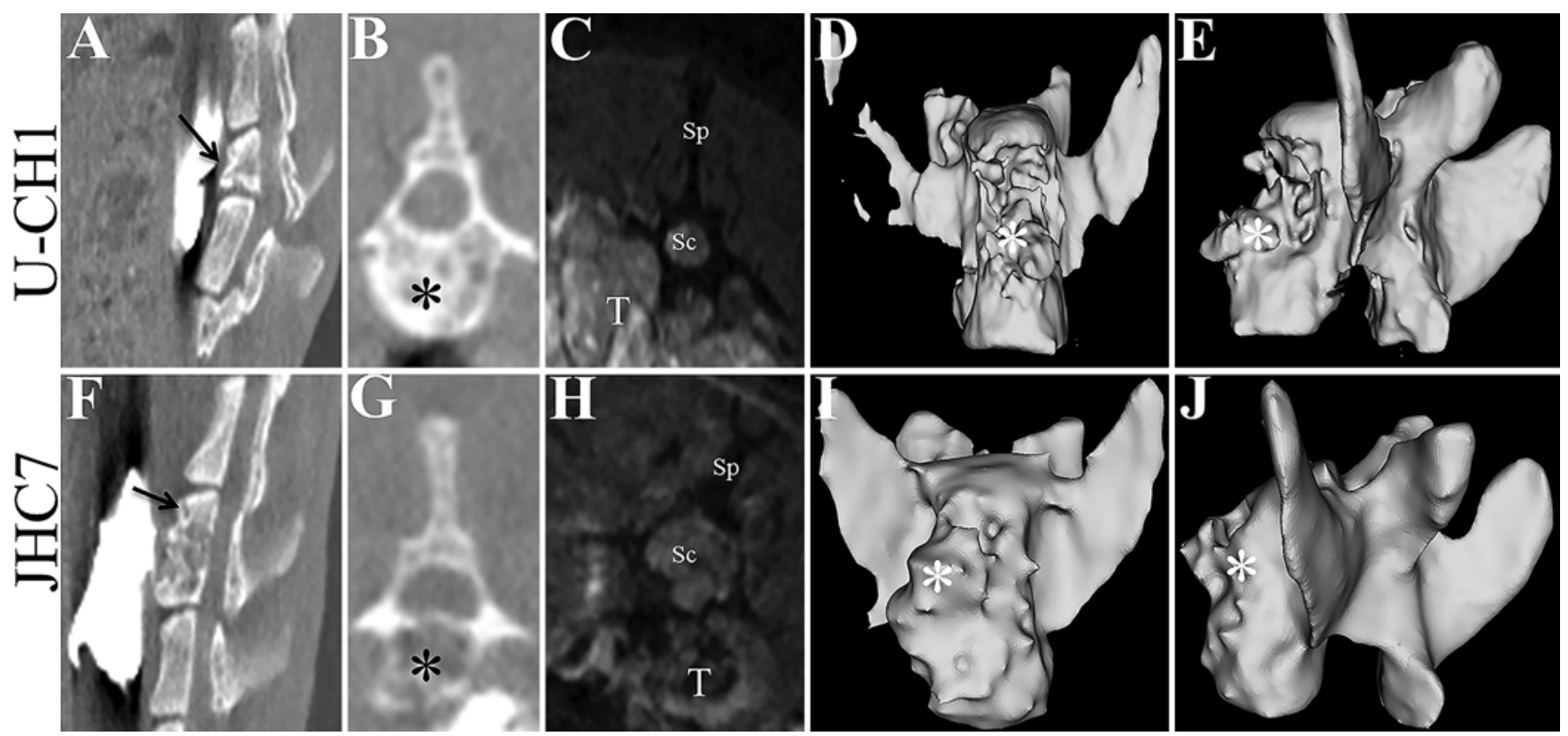

FIG. 3. Imaging of UCH1 and JHC7 spine chordomas. A and F: Sagittal views of the CBCT scans acquired 450 days after engraftment show tumor growth at the L-5 level in rats implanted with $\mathrm{UCH} 1$ and $\mathrm{JHC}$. Lesions were identified in the L-5 vertebral body (black arrows) of tumor-engrafted rats. B and G: Axial scans show osteolytic lesions in the vertebral body (black asterisks) in both groups. The polymer used to seal the bone cavity after tumor implantation is visible below the vertebral body as a big white solid mass. C and H: Axial T2-weighted MR images show a large lumbar UCH1 chordoma (C) arising from the L-5 vertebral body (T) and compressing the nerve roots without spinal cord compression in the UCH1 group. For the JHC7 chordoma $(\mathrm{H})$ arising from the L-5 vertebral body, the image demonstrates that the mass extends toward the lateral spinous process (T); there is a significant extension of the mass that is deforming the spinal cord and compressing the dorsal nerve roots. $\mathrm{D}$ and $\mathrm{E}$, I and J: 3D reconstructions of the nanoScan PET/CT imaging performed 545 days after engraftment. Anterior ( $D$ and $I$ ) and lateral ( $E$ and $J$ ) views of the affected L-5 lateral spinous process and vertebral bodies show bone damage due to tumor osteolysis in the UCH1 vertebral body (white asterisks) and bone overgrowth in the JHC7 vertebral body (white asterisks). $\mathrm{Sc}=$ spinal cord; $\mathrm{Sp}=$ spinous process; $\mathrm{T}=$ tumor.

bral body of L-5 in a honeycomb pattern (Fig. 3B, black asterisk). In the JHC7 tumor-engrafted rats, nanoScan PET/CT (Fig. 3D, E, I, and J) showed an increase in bone formation on the periphery of the L-5 vertebral body (Fig. 3I and J, white asterisks). The UCH1 tumor-engrafted rats showed lytic lesions in the vertebral body and spinous processes (Fig. 3D and E, white asterisks).

\section{Human Orthotopic Chordoma Growth Affected Locomo- tion, Allodynia, and Thermal Sensation in Rats}

After confirmation of the presence of tumors in the vertebral body, motor function was evaluated on Day 530 in tumor-engrafted rats. Kinematic analysis of unrestrained gait at the end of the study showed gait disturbances in the hindlimbs in both groups of tumor-engrafted rats compared with rats in the sham and naïve groups (Fig. 4A-F).

The step length and duration were increased in UCH1 tumor-engrafted rats by approximately $327.27 \%$ and $190 \%$, respectively. JHC7 tumor-engrafted rats showed similar significant increases of $381.81 \%$ and $153.33 \%(\mathrm{p}<$ 0.05 ) in step length and duration, respectively (Fig. 4C and $D)$. In addition, a significant difference $(\mathrm{p}=0.0012)$ was observed in the ankle angle of UCH1 rats during unrestrained gait locomotion (Supplementary Fig. 1). The pendulum-like leg movements during gait strides decreased significantly $(\mathrm{p}<0.001)$ during the flexion $(69.81 \%)$ and extension $(92.45 \%)$ phases of UCH1 tumor-engrafted rats, but no significant changes were observed in JHC7 tumorengrafted rats (Fig. 4E). Additionally, tumor-engrafted rats in both groups had a significant increase $(\mathrm{p}<0.001)$ in the raising (UCH1 $357.14 \%$ and $\mathrm{JHC} 7410.71 \%$ ) and return (UCH1 $440 \%$ and JHC7 460\%) phases of the hindlimb (Fig. 4F).

In the open field test, it was observed that during the 5 -minute period of analysis, tumor-engrafted rats had a significant $(p<0.05)$ decrease in exploratory behavior. The total distance traveled by the UCH1 tumor-engrafted rats was decreased by $61 \%$ and $66 \%$ and the total distance traveled by the JHC7 group was decreased by $23 \%$ and $31 \%$ compared with the control and sham groups, respectively (Fig. 5A). Similar results were observed in the total mean speed for the UCH1 rats (decreased by $68 \%$ compared with the control group and 69\% compared with the sham group); JHC7-engrafted rats showed similar reductions in the total mean speed of $69 \%$ and $72 \%$ compared with the control and sham groups, respectively (Fig. 5B).

Furthermore, the level of algesia in response to mechanical and thermal stimuli was evaluated in the tumorengrafted, control, and sham rats. During mechanical stimulation of paw withdrawal, a significant increase in the force needed to induce a response from the tumorengrafted rats compared with control rats was observed (UCH1 198\% and JHC7 203\%; p < 0.001). These results could indicate that tumor-engrafted rats have mechanical 

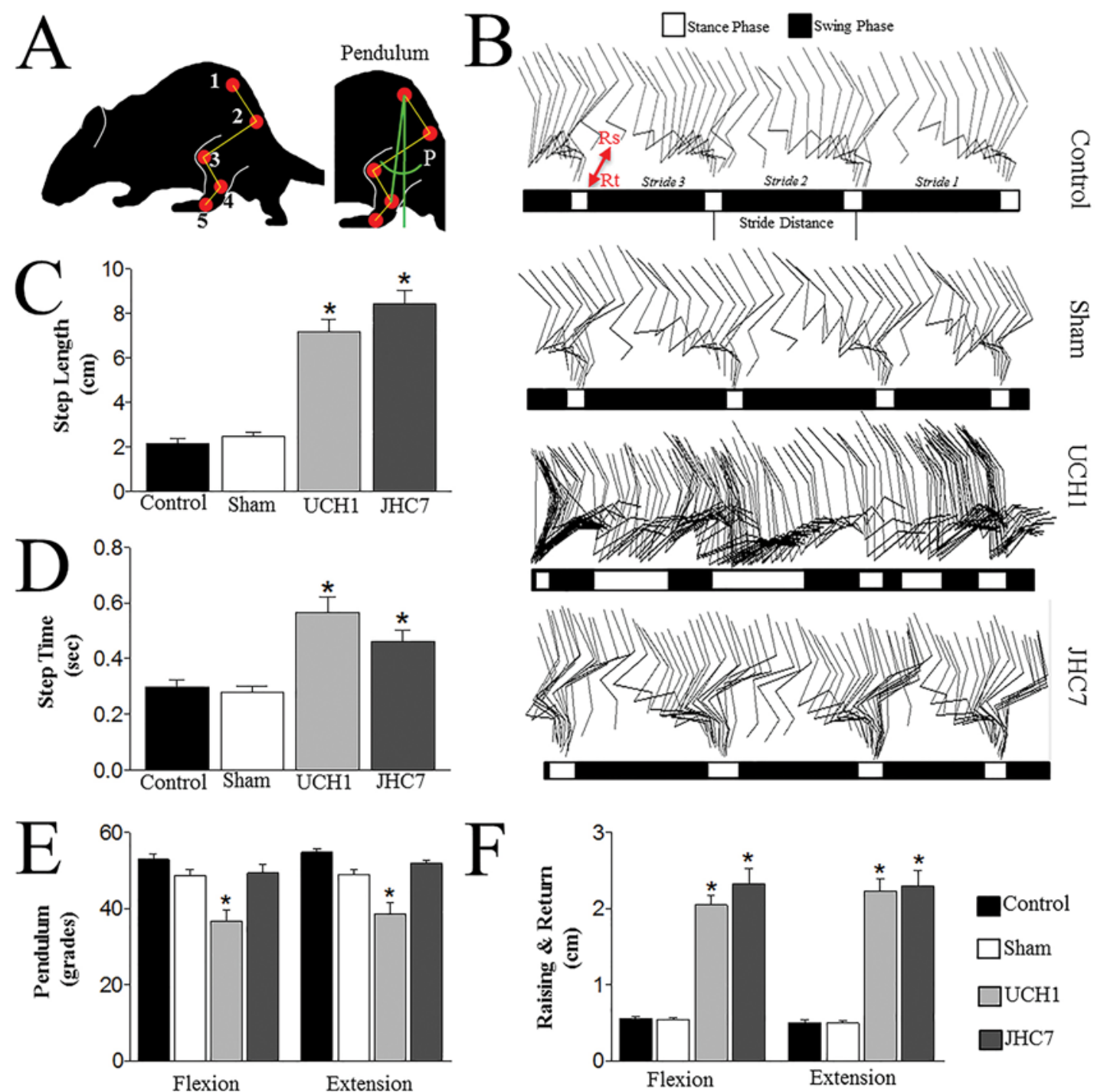

FIG. 4. Effects of human orthotopic chordoma in rats on hindlimb gait locomotion. A: Anatomical reference points $(1=$ iliac crest; 2 = great trochanter; 3 = knee; 4 = lateral malleolus; 5 = metatarsal-phalanx joint) used in the left hindlimb joints to evaluate kinematic parameters and pendulum-like movements $(P)$. B: The sequences of the hindlimb movements are shown in stick figures. Each panel represents 3 consecutive stride cycles in a graphic representation from the control, sham, UCH1, and JHC7 groups. The groups suffered from extended periods of stance (white) and swing (black) phases. C and D: Step length and step duration were evaluated on Day 530 in UCH1 and JHC7 tumor-engrafted rats. Data are presented as the mean $\pm \mathrm{SEM} ;{ }^{*} p<0.05$. Changes in the pendulum-like movement (E) and in the raising (Rs) and return (Rt) of the left hindlimb (F) occurred during flexion and extension in the unrestrained gait of rats. Data are presented as the mean $\pm S E M ;{ }^{*} p<0.0001$. Figure is available in color online only.

hypoalgesia compared with control rats (Fig. 5C). In contrast, tumor-engrafted rats showed a faster response (paw withdrawal) to a constant radiant heat stimulus $\left(50^{\circ} \mathrm{C}\right)$ than control rats (UCH1 144\% and JHC7 152\%), indicating thermal hyperalgesia (Fig. 5D). Sham rats showed no significant differences compared with naïve controls.

\section{Discussion}

The devastating nature of chordomas and our poor understanding of the biology necessitate the establishment of a reliable and clinically suitable animal model. The only orthotopic animal model of chordoma that has been reported uses zebrafish; ${ }^{6,12}$ however, the use of a nonmammal system is not ideal for representing the complexity of human disease. Other subcutaneous xenograft chordoma models derived from human tissues have been used to produce rodent tumors; however, they do not replicate the human disease in terms of neurological function, largely due to the ectopic location of tumors (hence a lack of influence on nociception and locomotion of the gait). ${ }^{9,19,21,23,40} \mathrm{In}$ this study, we sought to develop and evaluate an orthotopic animal model involving human-derived chordoma growing in the L-5 vertebral body of immunocompromised rats. With progressive growth, these tumors affected the locomotion and nociceptive behavior of rats as well as the bone anatomy, based on imaging evidence. Thus, this model clinically and radiographically represents the human condition of spinal chordoma. 

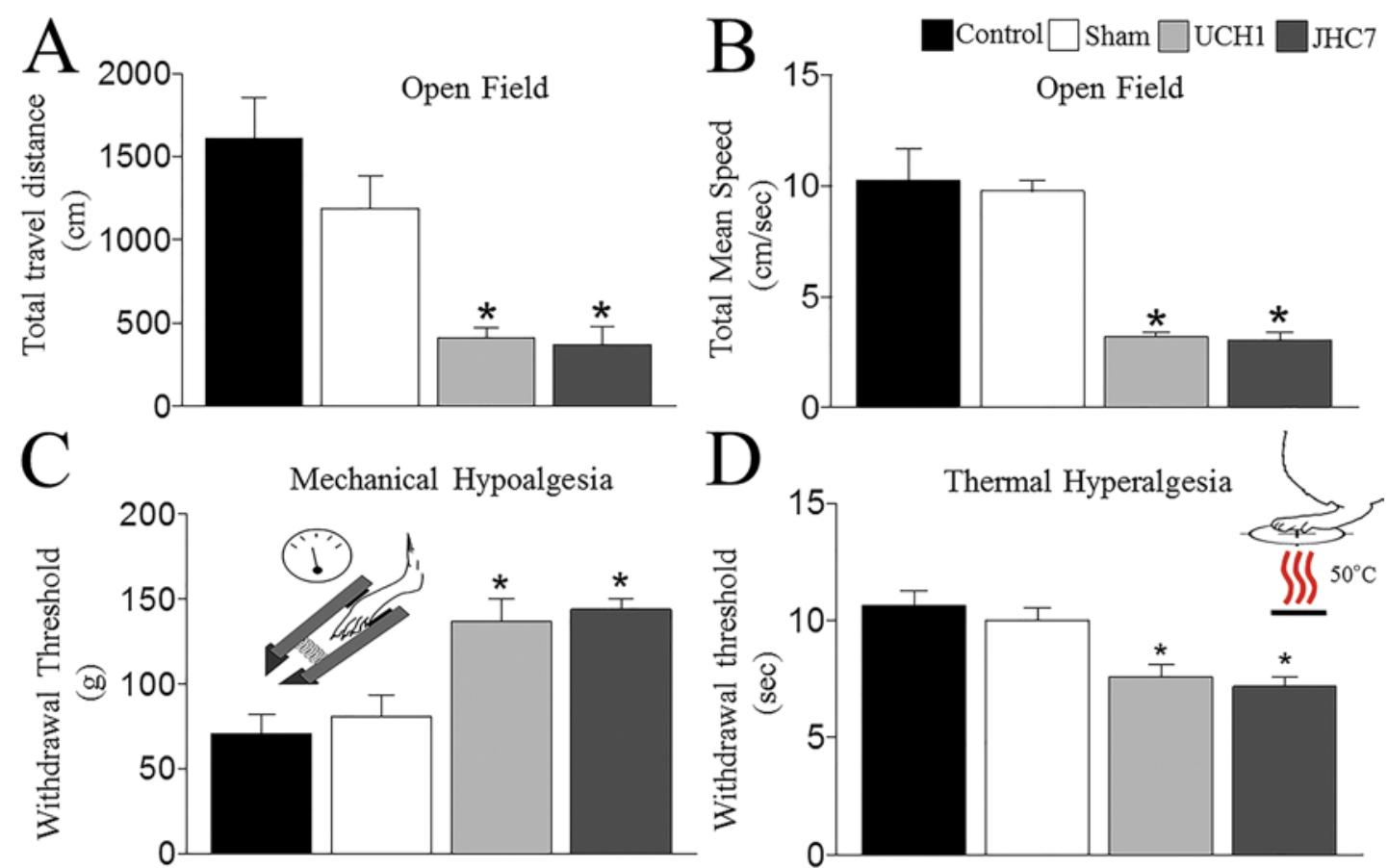

FIG. 5. Effects of intraspinal human chordoma on locomotion and nociceptive response in tumor-engrafted rats. Traveled distance $(\mathrm{cm})(\mathbf{A})$ and mean speed $(\mathrm{cm} / \mathrm{sec})(B)$ were reduced in rats engrafted with chordoma. Changes in the nociceptive response due to a mechanical (C) and thermal (D) stimulus showed opposite effects of intraspinal chordoma. Data are presented as the mean \pm $\mathrm{SEM} ;{ }^{*} p<0.05$. Figure is available in color online only.

\section{Tumor Growth}

Chordomas are thought to arise from remnants of the primitive notochord ${ }^{46}$ and are known to be locally aggressive and relatively unresponsive to chemotherapy and radiation. ${ }^{4,11,13,33}$ They exhibit highly infiltrative growth and are often lobulated by septa of connective tissue. In our preclinical model, an overall $68 \%$ of tumor-engrafted rats developed chordoma in the L-5 vertebral body within approximately 1.5 years, mimicking the slow-growth tumor pattern observed in patients with chordoma. The vertebral body and the lateral spinous processes in some of the UCH1 and JHC7 rats showed lytic lesions, as demonstrated by CBCT and the reconstructed nanoScan PET/CT images (Fig. 3). No other vertebral bodies were found to be affected. This is consistent with clinical reports where chordomas invade and destroy a single vertebral body without affecting the surrounding ones. ${ }^{10}$ Two rats in the UCH1 group presented a very large tumor with soft-tissue involvement. This is consistent with the description of the pathology of recurrent chordomas, in which these malignancies infiltrate muscle and soft tissue, especially in cases of tumors that have received radiotherapy. ${ }^{44}$

Delank et al..$^{10}$ reported that the clinical symptomatology of human spinal chordoma is determined by the location and expansion of the tumor as well as the tumor's relation to adjacent anatomical structures. The origin of the tumor is centrally based in the vertebral body, which is possibly explained by the embryological development of the notochord. The tumor location and characteristics are responsible for the fact that there often is a long latency between the development of the tumor and the diagnosis in spinal chordomas (an average of 1.5 years). ${ }^{10,35}$
These findings are consistent and accurate with our chordoma model, where we engrafted the tumor in a central location. The tumor from the 2 chordoma cell lines grew and infiltrated the bony tissue in approximately 1.5 years. Spinal chordomas often spread through the bloodstream, ${ }^{51}$ with distant metastatic rates differing based on location. The most common sites of metastases are lung and lymph nodes. Liver and bone metastases are also frequent. ${ }^{28,37,45,51}$ In our UCH1 or JHC7 tumor-engrafted rats, there were no signs of metastasis in other organs at the completion of the study (unpublished data); this is consistent with the lack of metastasis observed in patients., ${ }^{2,16}$

\section{Tumor Histology}

Gross consistency of chordoma is macroscopically described as semiliquid, soft, green, and transparent. ${ }^{19,29,41,44}$ Chordomas frequently contain focal calcifications, ossifications, hemorrhagic areas, necrosis, and cyst formations. Microscopic examination shows the pathognomonic histological characteristics of these tumors, which is the presence of physaliferous cells (Fig. 2C), described as vacuolated cells that contain intracytoplasmic mucus. These cells are separated by fibrous septa into lobules and surrounded by a basophilic extracellular matrix rich in mucin and glycogen. ${ }^{8,43}$ Our results showed that chordomas engrafted in immunocompromised rats retained the morphological and histological characteristics of human chordoma, indicative that a chordoma developed in the vertebral body (Figs. 1 and 2), as confirmed by a blinded pathologist. Immunostaining of UCH1 tumor for cytokeratins was positive in the tumor cells and negative in the connective tissue. In the 
$\mathrm{UCH} 1$ and JHC7 groups, tumor was present in the trabeculae of the bone, showing the typical multinodular arrangement (Fig. 1C). Chordoma histology was better preserved in the JHC7 group; physaliferous cells were observed between the fibrous septa. Strong nuclear immunoreactivity for brachyury was confirmed in both tumors, with clearly negative immunoreactivity in the stroma (Fig. 2C).

\section{Imaging Characteristics}

The imaging characteristics of the chordomas in our model showed radiographic features that are similar to those observed in patients. The vertebrae appear with irregular anatomy because of the expansive destruction induced by a slow-growing soft-tissue lesion, remodeling and reactivating bone formation. ${ }^{46}$ According to Sundaresan, ${ }^{44}$ a soft-tissue mass anterior to the involved vertebrae is the most important radiological finding and shows that paravertebral tissues are more affected than osseous tissues, similar to what we observed in the UCH1 tumor-engrafted rats. The vertebral lesions are characterized primarily by destructive changes involving the body, often with a surrounding area of reactive sclerosis. Involvement of adjacent vertebral bodies is a frequent finding in chordomas, but in our model we found that only 1 vertebra was involved.

In our CBCT images from UCH1 and JHC7 tumorengrafted rats, we observed some areas of calcifications, sclerotic changes, and bony destruction (Fig. 3A, B, F, and $\mathrm{G})$. Contrast-enhanced MRI was used to confirm the spinal chordoma. It is well known that MRI is the gold standard for diagnostic purposes; chordoma is commonly described as iso- or hypointense on T1-weighted images and moderately hyperintense on T2-weighted images. T1 with contrast shows a characteristic heterogeneous enhancement that is classically described as honeycombing. In our UCH1 and JHC7 tumor-engrafted-rats, we observed on T1- and T2-weighted MR images an iso-intensity and high-intensity signal, respectively, as shown in Fig. 3C and $\mathrm{H}$.

\section{Clinical Evaluation}

Patients with spinal chordomas more commonly present with paresthesias and pain; nevertheless, neurological symptoms are seen in later stages of the disease. ${ }^{15}$ The symptoms are closely related to localization and growth rate of the tumor, as well as the compression of nerve roots, spinal cord, paravertebral tissues, and surrounding anatomical structures by the tumor. Gallia et al. ${ }^{15}$ reported that a 52-year-old patient with a large sacral chordoma presented with a 15-year history of sacral pain and a 4-year history of constipation. On physical examination, they observed a mass protruding through the right side of the sacrum and the right sacroiliac joint region.

These findings are consistent with 1 of our rats with a UCH1 chordoma, which was found to have a mass protruding through the left side of the sacrum at the same level as that demonstrated clinically. Necropsy of the lower spine of the rat revealed a large tumor invading the abdominal cavity, similar to the patient described by Gallia et al. In their study, the patient's MR image revealed a large tumor originating from the S2-3 junction, extending posteriorly, laterally involving the right sacroiliac joint, and anteriorly filling the entire pelvic cavity. The MR image of the UCH1-bearing rat revealed a large tumor originating from the L-5 vertebral body and extending posteriorly to the sacrococcygeal spine (Fig. 3C). The neurological evaluation of the patient with a large chordoma demonstrated isolated plantar flexor weakness bilaterally. The patient suffered no sensory loss in the lower extremities or perianal region. The patient in this study exhibited a normal gait according to the authors,,${ }^{15}$ but other patients with chordoma present with abnormal gait or gait difficulties. ${ }^{16,30}$

Gait kinematic analysis is a useful tool to evaluate the motor function of the hindlimbs in tumor-engrafted rats..$^{38}$ The stride length of the left hindlimb was affected 530 days after tumor engraftment; the right hindlimb had a compensatory role by supporting more weight during locomotion. Interestingly, compared with the control and sham groups, the tumor-engrafted rats exhibited increased stride lengths in the left hindlimb due to the increased length in the stance and swing phases. In patients, the majority of abnormalities of pathological gait are observed during the stance phase, when all body weight is supported by 1 leg. It is during this phase that pain, muscle weakness, and joint abnormalities produce their predominant effects.

In our chordoma model, we observed abnormalities in the swing and stance phases in tumor-engrafted rats. In our control rats, during the stance phase, the dorsiflexion in the ankle reached its peak near the midstance phase, and then plantar flexion was observed until the end of this phase. In our UCH1 tumor-engrafted rats, the dorsiflexion in the ankle was significantly decreased $(p=0.0012)$ (Supplementary Fig. 1) and the plantar stepping was absent (data unpublished). Raising and return of the hindlimb was increased in all of the tumor-engrafted rats. This could be explained by the decrease in the dorsiflexion of the knee and ankle due to muscle weakness as a consequence of compression of the sciatic nerve by the chordoma.

Gait kinematics are well characterized in intact rats, ${ }^{18,25}$ but there is a lack of studies on pathologic gait due to chordomas in rodents. In this study, we analyzed sagittal walking of rats in a corridor. The UCH1 group showed an exaggerated hip and knee flexion and diminished flexionextension of the ankle during gait. This could have been due to weakness of the dorsi-flexor muscles of the leg (caused by compression of the sciatic nerve by the tumor, resulting in motor neuron degeneration and consequent loss of innervation and strength). These abnormalities were observed during the swing phase (Fig. 4). The effects of compression by tumor in our model can be compared with the effects caused by direct mechanical compression of the sciatic nerve, where the muscles below the knee present weakness while walking. ${ }^{1}$

Lesions in the spine due to chordoma frequently cause motor changes in patients due to spinal cord or root compression, but paraplegia rarely occurs as a complication. Gait locomotion analysis in the tumor-engrafted rats showed some joint disturbances, that is, the sacroiliac joint in the UCH1 and JHC7 tumor-engrafted rats showed a slight (nonsignificant) increase in displacement during the flexion-extension phases. The same results were observed in the knee and ankle joints (data not shown) except for the 
UCH1 tumor-engrafted rats, which presented a significant increase $(p<0.05)$ of $83.33 \%$ in the flexion and $82.45 \%$ in the extension of the ankle when compared with the control group. Loss of plantar flexion and dropped foot are the most common symptoms in patients with tumors affecting the L4-5 vertebrae, which is correlated with nerve root tumor involvement..$^{53}$

The methods used to evaluate pain behavior in our model are well established and reliable quantitative measures of pain hypersensitivity in models of neuropathic pain. Currently, there are no techniques available to evaluate pain derived from spinal tumors in rodents. We evaluated the nociceptive response to mechanical and thermal stimuli, because the major cutaneous receptor types are found in the paw skin. ${ }^{22}$ Our results are similar to the ones found in rats with neuropathic pain due to sciatic nerve constriction. ${ }^{31}$ The tumor-engrafted rats showed thermal and mechanical hyperalgesia. This could be explained by the compression produced by the tumor in the dorsal nerve roots, as observed in the MR images of the UCH1 and JHC7 tumor-engrafted rats (Fig. 3C and H).

Macroscopic analysis revealed that the tumor extended significantly into the paravertebral area, compressing the nerve roots at the L-5 vertebra. Patients with lumbar vertebra chordoma can suffer from neuropathic pain and radicular pain during the course of their illness, years before diagnosis of the tumor. Pain in the lower back or coccygeal region is the most frequent symptom ${ }^{3,5,8,15,16}$ of patients with tumors located in these areas. Thus, our model underscores the need to use orthotopic tumor models to emulate the functional deficits presented by patients with this disease. Through the use of clinically relevant tumor models, we can gain further insights into the biology of these rare malignancies.

With this model, we have characterized a human-derived chordoma in the spine of an immunocompromised rat. Thus, we have created a platform that may accurately represent the human condition with respect to tumor growth, histology, imaging, and clinical presentation. Given the challenges of treating chordoma in the human population, we hope to further refine this model and use it as a tool to study the biology of these rare but lethal tumors, as well as to evaluate the efficacy of various existing and novel treatment modalities directed at improved local control and possible cure for chordoma. Nevertheless, our group understands the limitations of this model, as well as the optimization required to make this a standardized model for the study of chordoma. The latency of symptom appearance after engraftment, although it recapitulates the clinical scenario, poses a challenge to making this a simple model for the study of chordoma biology and its therapeutic implications.

Our group and others have used patient-derived xenografts, $, 21,40,41$ which exhibit faster growth rates that could shorten the length of time before the appearance of symptoms. Previously we observed that subsequent implantation of subcutaneous grafts from JHC7 became more aggressive with each passage, increasing its growth rate substantially. ${ }^{3}$ The aforementioned techniques would help increase the tumor establishment rate, which we acknowledge is not optimal. There is a concern whether xenografts established from cell lines derived from patients will have an altered biology compared with xenografts derived directly from intraoperative tissue. Nonetheless, the former confers the benefit of allowing for the performance of in vitro studies, which can lead to novel discoveries in chordoma biology, as well as large drug screening studies.

In summary, this chordoma model constitutes an advance for the study of this malignant disease, setting the basis for a broader study to improve our understating of the neurological deficits that occur when chordoma affects the spine. Understanding the entire pathophysiology of the disease will help tailor holistic therapeutic strategies that might lead to decreases in morbidity and mortality in patients with spine chordoma.

\section{Conclusions}

In this study, we developed an orthotopic rat model of spine chordoma using human cells. Tumor-engrafted rats were evaluated for neurological impairment and underwent histopathological analyses to determine the extent of disease. Tumor growth affected the locomotion and pain sensation of tumor-engrafted rodents in ways that mimic deficits present in humans afflicted with chordoma. Our intraspinal preclinical model represents a reliable method to evaluate experimental therapeutic approaches to human chordoma in rats.

\section{References}

1. Anand P, Mathangi DC, Mathew J, Namasivayam A, Suresh BR: Behavioral analysis after sciatic nerve compression in albino rats. Ann Neurosci 18:37-43, 2011

2. Bergh P, Kindblom LG, Gunterberg B, Remotti F, Ryd W, Meis-Kindblom JM: Prognostic factors in chordoma of the sacrum and mobile spine: a study of 39 patients. Cancer 88:2122-2134, 2000

3. Bjornsson J, Wold LE, Ebersold MJ, Laws ER: Chordoma of the mobile spine. A clinicopathologic analysis of 40 patients. Cancer 71:735-740, 1993

4. Boriani S, Bandiera S, Biagini R, Bacchini P, Boriani L, Cappuccio M, et al: Chordoma of the mobile spine: fifty years of experience. Spine (Phila Pa 1976) 31:493-503, 2006

5. Boriani S, Saravanja D, Yamada Y, Varga PP, Biagini R, Fisher CG: Challenges of local recurrence and cure in low grade malignant tumors of the spine. Spine (Phila Pa 1976) 34 (22 Suppl):S48-S57, 2009

6. Burger A, Vasilyev A, Tomar R, Selig MK, Nielsen GP, Peterson RT, et al: A zebrafish model of chordoma initiated by notochord-driven expression of HRASV12. Dis Model Mech 7:907-913, 2014

7. Chugh R, Tawbi H, Lucas DR, Biermann JS, Schuetze SM, Baker LH: Chordoma: the nonsarcoma primary bone tumor. Oncologist 12:1344-1350, 2007

8. Dahlin DC, MacCarty CS: Chordoma. Cancer 5:1170-1178, 1952

9. Davies JM, Robinson AE, Cowdrey C, Mummaneni PV, Ducker GS, Shokat KM, et al: Generation of a patient-derived chordoma xenograft and characterization of the phosphoproteome in a recurrent chordoma. J Neurosurg 120:331-336, 2014

10. Delank KS, Kriegsmann J, Drees P, Eckardt A, Eysel P: Metastasizing chordoma of the lumbar spine. Eur Spine $\mathbf{J}$ 11:167-171, 2002

11. Ferraresi V, Nuzzo C, Zoccali C, Marandino F, Vidiri A, Salducca N, et al: Chordoma: clinical characteristics, man- 
agement and prognosis of a case series of 25 patients. BMC Cancer 10:22, 2010

12. Ferrari L, Pistocchi A, Libera L, Boari N, Mortini P, Bellipanni G, et al: FAS/FASL are dysregulated in chordoma and their loss-of-function impairs zebrafish notochord formation. Oncotarget 5:5712-5724, 2014

13. Fuji H, Nakasu Y, Ishida Y, Horiguchi S, Mitsuya K, Kashiwagi $\mathrm{H}$, et al: Feasibility of proton beam therapy for chordoma and chondrosarcoma of the skull base. Skull Base 21:201-206, 2011

14. Fujita T, Kawahara N, Matsumoto T, Tomita K: Chordoma in the cervical spine managed with en bloc excision. Spine (Phila Pa 1976) 24:1848-1851, 1999

15. Gallia GL, Haque R, Garonzik I, Witham TF, Khavkin YA, Wolinsky JP, et al: Spinal pelvic reconstruction after total sacrectomy for en bloc resection of a giant sacral chordoma. Technical note. J Neurosurg Spine 3:501-506, 2005

16. Gokaslan ZL, Zadnik PL, Sciubba DM, Germscheid N, Goodwin CR, Wolinsky JP, et al: Mobile spine chordoma: results of 166 patients from the AOSpine Knowledge Forum Tumor database. J Neurosurg Spine 24:644-651, 2016

17. Hargreaves K, Dubner R, Brown F, Flores C, Joris J: A new and sensitive method for measuring thermal nociception in cutaneous hyperalgesia. Pain 32:77-88, 1988

18. Hruska RE, Kennedy S, Silbergeld EK: Quantitative aspects of normal locomotion in rats. Life Sci 25:171-179, 1979

19. Hsu W, Mohyeldin A, Shah SR, ap Rhys CM, Johnson LF, Sedora-Roman NI, et al: Generation of chordoma cell line JHC7 and the identification of Brachyury as a novel molecular target. J Neurosurg 115:760-769, 2011

20. Jeys L, Gibbins R, Evans G, Grimer R: Sacral chordoma: a diagnosis not to be sat on? Int Orthop 32:269-272, 2008

21. Karikari IO, Gilchrist CL, Jing L, Alcorta DA, Chen J, Richardson WJ, et al: Molecular characterization of chordoma xenografts generated from a novel primary chordoma cell source and two chordoma cell lines. J Neurosurg Spine 21:386-393, 2014

22. Leem JW, Willis WD, Chung JM: Cutaneous sensory receptors in the rat foot. J Neurophysiol 69:1684-1699, 1993

23. Liu X, Nielsen GP, Rosenberg AE, Waterman PR, Yang W, Choy E, et al: Establishment and characterization of a novel chordoma cell line: CH22. J Orthop Res 30:1666-1673, 2012

24. Luque Contreras D, Jiménez Estrada I, Martínez Fong D, Segura B, Guadarrama JC, Paniagua Sierra R, et al: Hindlimb claudication reflects impaired nitric oxide-dependent revascularization after ischemia. Vascul Pharmacol 46:10-15, 2007

25. Majczyński H, Maleszak K, Górska T, Sławińska U: Comparison of two methods for quantitative assessment of unrestrained locomotion in the rat. J Neurosci Methods 163:197-207, 2007

26. Mantha A, Legnani FG, Bagley CA, Gallia GL, Garonzik I, Pradilla G, et al: A novel rat model for the study of intraosseous metastatic spine cancer. J Neurosurg Spine 2:303-307, 2005

27. McMaster ML, Goldstein AM, Bromley CM, Ishibe N, Parry DM: Chordoma: incidence and survival patterns in the United States, 1973-1995. Cancer Causes Control 12:1-11, 2001

28. McPherson CM, Suki D, McCutcheon IE, Gokaslan ZL, Rhines LD, Mendel E: Metastatic disease from spinal chordoma: a 10-year experience. J Neurosurg Spine 5:277-280, 2006

29. Mohindra S, Kapoor A, Kursa GK, Mohindra S, Saikia U: Apoplexy in an intradural clival chordoma causing intraventricular bleed. Surg Neurol Int 7 (Suppl 1):S17-S19, 2016

30. Nishiguchi T, Mochizuki K, Tsujio T, Nishita T, Inoue Y: Lumbar vertebral chordoma arising from an intraosseous benign notochordal cell tumour: radiological findings and histopathological description with a good clinical outcome. Br J Radiol 83:e49-e53, 2010

31. Obata K, Yamanaka H, Fukuoka T, Yi D, Tokunaga A, Hashimoto N, et al: Contribution of injured and uninjured dorsal root ganglion neurons to pain behavior and the changes in gene expression following chronic constriction injury of the sciatic nerve in rats. Pain 101:65-77, 2003

32. Ordonez AA, Pokkali S, DeMarco VP, Klunk M, Mease RC, Foss CA, et al: Radioiodinated DPA-713 imaging correlates with bactericidal activity of tuberculosis treatments in mice. Antimicrob Agents Chemother 59:642-649, 2015

33. Park L, Delaney TF, Liebsch NJ, Hornicek FJ, Goldberg S, Mankin H, et al: Sacral chordomas: Impact of high-dose proton/photon-beam radiation therapy combined with or without surgery for primary versus recurrent tumor. Int J Radiat Oncol Biol Phys 65:1514-1521, 2006

34. Razgado-Hernandez LF, Espadas-Alvarez AJ, ReynaVelazquez P, Sierra-Sanchez A, Anaya-Martinez V, JimenezEstrada I, et al: The transfection of BDNF to dopamine neurons potentiates the effect of dopamine D3 receptor agonist recovering the striatal innervation, dendritic spines and motor behavior in an aged rat model of Parkinson's disease. PLoS One 10:e0117391, 2015

35. Rosell Pradas J, Ruiz Morales M, Guerrero Fernández JA, Vara-Thorbeck R: [Sacrococcygeal chordoma. Results of radical operation.] Zentralbl Chir 116:1253-1257, 1991 (Ger)

36. Rossignol S, Chau C, Brustein E, Bélanger M, Barbeau H, Drew T: Locomotor capacities after complete and partial lesions of the spinal cord. Acta Neurobiol Exp (Warsz) 56:449-463, 1996

37. Safari M, Khoshnevisan A: An overview of the role of cancer stem cells in spine tumors with a special focus on chordoma. World J Stem Cells 6:53-64, 2014

38. Sarabia-Estrada R, Zadnik PL, Molina CA, Jimenez-Estrada I, Groves ML, Gokaslan ZL, et al: A rat model of metastatic spinal cord compression using human prostate adenocarcinoma: histopathological and functional analysis. Spine J 13:1597-1606, 2013

39. Scheil S, Brüderlein S, Liehr T, Starke H, Herms J, Schulte $\mathrm{M}$, et al: Genome-wide analysis of sixteen chordomas by comparative genomic hybridization and cytogenetics of the first human chordoma cell line, U-CH1. Genes Chromosomes Cancer 32:203-211, 2001

40. Siu IM, Ruzevick J, Zhao Q, Connis N, Jiao Y, Bettegowda C, et al: Erlotinib inhibits growth of a patient-derived chordoma xenograft. PLoS One 8:e78895, 2013

41. Siu IM, Salmasi V, Orr BA, Zhao Q, Binder ZA, Tran C, et al: Establishment and characterization of a primary human chordoma xenograft model. J Neurosurg 116:801-809, 2012

42. Stacchiotti S, Casali PG, Lo Vullo S, Mariani L, Palassini E, Mercuri M, et al: Chordoma of the mobile spine and sacrum: a retrospective analysis of a series of patients surgically treated at two referral centers. Ann Surg Oncol 17:211-219, 2010

43. Sun X, Hornicek F, Schwab JH: Chordoma: an update on the pathophysiology and molecular mechanisms. Curr Rev Musculoskelet Med 8:344-352, 2015

44. Sundaresan N: Chordomas. Clin Orthop Relat Res (204):135-142, 1986

45. Tranesh G, Nassar A: Metastatic chordoma: a diagnostic challenge on fine needle aspiration. Case Rep Pathol 2016:2187290, 2016

46. Ulich TR, Mirra JM: Ecchordosis physaliphora vertebralis. Clin Orthop Relat Res (163):282-289, 1982

47. Vivancos GG, Verri WA Jr, Cunha TM, Schivo IR, Parada CA, Cunha FQ, et al: An electronic pressure-meter nociception paw test for rats. Braz J Med Biol Res 37:391-399, 2004

48. von Witzleben A, Goerttler LT, Lennerz J, Weissinger S, Ko- 
rnmann M, Mayer-Steinacker R, et al: In chordoma, metastasis, recurrences, Ki-67 index, and a matrix-poor phenotype are associated with patients' shorter overall survival. Eur Spine J:4016-4024, 2016

49. Wong J, Armour E, Kazanzides P, Iordachita I, Tryggestad E, Deng H, et al: High-resolution, small animal radiation research platform with x-ray tomographic guidance capabilities. Int J Radiat Oncol Biol Phys 71:1591-1599, 2008

50. Yang Y, Niu X, Li L, Ding Y: The chordoma arised from ilium: a rare case report. J Bone Oncol 4:103-106, 2015

51. Young VA, Curtis KM, Temple HT, Eismont FJ, DeLaney TF, Hornicek FJ: Characteristics and patterns of metastatic disease from chordoma. Sarcoma 2015:517657, 2015

52. Zadnik P, Sarabia-Estrada R, Groves ML, Molina C, Jackson C, McCarthy E, et al: A novel animal model of human breast cancer metastasis to the spine: a pilot study using intracardiac injection and luciferase-expressing cells. J Neurosurg Spine 18:217-225, 2013

53. Zoccali C, Skoch J, Patel AS, Walter CM, Maykowski P, Baaj AA: Residual neurological function after sacral root resection during en-bloc sacrectomy: a systematic review. Eur Spine J 25:3925-3931, 2016

\section{Disclosures}

Dr. Gokaslan has direct stock ownership in Spinal Kinetics. Dr. Goodwin is a United Negro College Fund-Merck Science Initiative postdoctoral fellow and received support from the Burroughs Wellcome Fund and Johns Hopkins Neurosurgery Pain Research Institute. Dr. Jain received clinical or research support for the study described (including equipment or material) from Gilead Sciences. Dr. Sarabia-Estrada received support from AOSpine North America and the Johns Hopkins Neurosurgery Pain Research Institute. Dr. Sciubba received support from the Johns
Hopkins Neurosurgery Pain Research Institute and is a consultant for Medtronic, DePuy Synthes, Globus, Orthofix, and Stryker.

\section{Author Contributions}

Conception and design: Sciubba, Sarabia-Estrada, Ruiz-Valls, Shah, Gokaslan, Quinones-Hinojosa. Acquisition of data: SarabiaEstrada, Ruiz-Valls, Shah, Ordonez, Velarde, Li, Phillips, Jain. Analysis and interpretation of data: Sciubba, Sarabia-Estrada, Ruiz-Valls, Shah, Ordonez, Rodriguez, Guerrero-Cazares, Jimenez-Estrada, Velarde, Jain, Gokaslan, Quinones-Hinojosa. Drafting the article: Sarabia-Estrada, Ruiz-Valls, Ahmed, Guerrero-Cazares, Jimenez-Estrada, Tyler. Critically revising the article: all authors. Reviewed submitted version of manuscript: all authors. Approved the final version of the manuscript on behalf of all authors: Sciubba. Statistical analysis: Sarabia-Estrada, RuizValls. Administrative/technical/material support: Phillips. Study supervision: Sciubba, Sarabia-Estrada, Ruiz-Valls.

\section{Supplemental Information \\ Online-Only Content}

Supplemental material is available with the online version of the article.

Supplementary Figure 1. https://thejns.org/doi/suppl/10.3171/ 2016.12.SPINE16917.

\section{Previous Presentations}

Portions of this work were presented at the Annual Meeting of the American Association of Cancer Research, Philadelphia, PA, April 18-22, 2015.

\section{Correspondence}

Daniel M. Sciubba, Department of Neurosurgery, Johns Hopkins University School of Medicine, 600 North Wolfe St., Meyer 5-185, Baltimore, MD 212087. email: dsciubb1@jhmi.edu. 